\title{
Climate Change: Anticipated Effects on High-Energy Laser Weapon Systems in Maritime Environments
}

\author{
Steven T. Fiorino, Robb M. Randall, Richard J. Bartell, and Adam D. Downs \\ Air Force Institute of Technology, Wright-Patterson Air Force Base, Ohio \\ Peter C. Chu And C. W. FAN \\ Naval Postgraduate School, Monterey, California
}

(Manuscript received 26 January 2010, in final form 12 August 2010)

\begin{abstract}
This study quantifies the potential impacts on ship-defense high-energy-laser (HEL) performance due to atmospheric effects in the marine boundary layer driven by recent observations and analysis of worldwide sea surface temperatures (SSTs). The atmospheric effects are defined using the worldwide probabilistic climatic database available in the High Energy Laser End-to-End Operational Simulation (HELEEOS) model, which includes an SST database for the period 1854-1997. A more recent worldwide sea surface temperature database was provided by the Naval Postgraduate School for the period 1990-2008. Mean differences and trends between the two SST databases are used to deduce possible climate change impacts on simulated maritime HEL engagements. The anticipated effects on HEL propagation performance are assessed at an operating wavelength of $1.0642 \mu \mathrm{m}$ across the world's oceans and mapped onto a $1^{\circ} \times 1^{\circ}$ grid. The scenario evaluated is near surface and nearly horizontal over a range of $5000 \mathrm{~m}$ in which anticipated clear-air maritime aerosols occur. Summer and winter scenarios are considered. In addition to realistic vertical profiles of molecular and aerosol absorption and scattering, correlated optical turbulence profiles in probabilistic (percentile) format are used.
\end{abstract}

\section{Introduction}

For the purpose of evaluating an expected impact of climate change on directed energy weapon (DEW) system performance, the Air Force Institute of Technology (AFIT) Center for Directed Energy (AFIT CDE) employed several modeling codes to simulate operating conditions. One of these codes, the High Energy Laser End-to-End Operational Simulation (HELEEOS), is perhaps the first DEW simulation package to fully incorporate a correlated, probabilistic climatological database. The infusion of such realistic atmospheric effects into the simulations allows HELEEOS to better assess variability and uncertainty in system performance arising from spatial, spectral, and temporal variations in operating conditions (Fiorino et al. 2008; Bartell et al. 2005). The current study quantifies the impacts on ship-defense high-energy-laser (HEL) performance due to atmospheric effects in the marine

Corresponding author address: Dr. Steven T. Fiorino, AFIT/ ENP, 2950 Hobson Way, Wright-Patterson AFB, OH 45433-7765. E-mail: steven.fiorino@afit.edu boundary layer driven by recent changes in worldwide sea surface temperature (SST) variations and trends. The baseline atmospheric effects are defined using the worldwide probabilistic climatic database available in the HELEEOS model, which includes an SST database for the period 1854-1997. The Naval Postgraduate School in Monterey, California, provided AFIT CDE with a more recent worldwide sea surface temperature database for the period 1990-2008. Mean differences and trends between the two SST databases are used to deduce possible climate change impacts on simulated maritime HEL engagements. The anticipated effects on HEL propagation performance are assessed at an operating wavelength of $1.0642 \mu \mathrm{m}$ across the world's oceans and mapped onto a $1^{\circ} \times 1^{\circ}$ worldwide grid. The scenario evaluated is near surface and nearly horizontal over a range of $5000 \mathrm{~m}$ in which anticipated clear-air maritime aerosols occur. Summer and winter scenarios are considered. In addition to realistic vertical profiles of molecular and aerosol absorption and scattering, correlated optical turbulence profiles in probabilistic (percentile) format are used. 


\section{a. Description of the HELEEOS model}

The AFIT-developed HELEEOS modeling program includes a fast-calculating, first-principles, worldwide surface-to-100 km, atmospheric propagation and characterization package. This package enables the creation of profiles of temperature, pressure, water vapor content, optical turbulence, atmospheric particulates, and hydrometeors as they relate to line-by-line layer transmission, path, and background radiance at wavelengths from the ultraviolet to radio frequencies. Physics-based cloud and precipitation characterizations are coupled with physically probable temperature and moisture vertical lapse rates to create realistic atmospheric boundary layer effects. HELEEOS characterizes maritime aerosol environments using the Advanced Navy Aerosol Model (ANAM) or various representations of maritime particulates from the Global Aerosol Dataset (GADS). In the lowest $50 \mathrm{~m}$, HELEEOS defines the maritime optical turbulence with the Navy Surface Layer Optical Turbulence (NSLOT) model.

The HELEEOS model enables the evaluation of uncertainty in near-infrared laser and microwave propagation by incorporating probabilistic climatological data on the parameters that drive most major atmospheric effects. Atmospheric parameters investigated, such as temperature, pressure, water vapor content, optical turbulence, and atmospheric particulates, are put into vertical profiles of data for highly specific modeling scenarios. Worldwide seasonal, diurnal, and geographical spatialtemporal variations in these parameters are organized into probability density function (PDF) databases using a variety of recently available resources to include the Extreme and Percentile Environmental Reference Tables (ExPERT; Squires et al. 1995), the Master Database for Optical Turbulence Research in Support of Airborne Laser (Bussey et al. 2000), the Global Aerosol Dataset (Koepke et al. 1997), and Air Force Weather Agency numerical weather forecasting data. GADS provides aerosol constituent number densities on a $5^{\circ} \times 5^{\circ}$ grid worldwide. ExPERT mapping software allows the HELEEOS operator to choose from specific site or regional upper-air data to characterize the correlated molecular absorption, aerosol absorption, and scattering by percentile. The PDF nature of the HELEEOS atmospheric effects package enables realistic probabilistic outcome analyses, which permit an estimation of the confidence in the calculated probability of effect. HELEEOS users can additionally access, display, and export the atmospheric data independent of an HEL engagement simulation (Fiorino et al. 2006). Molecular scattering is computed based on Rayleigh theory. Molecular absorption effects are computed for the top 13 absorbing species using line strength information from the High-Resolution Transmission (HITRAN) 2004 database (Rothman et al. 2005) in conjunction with a community standard molecular absorption continuum code. Aerosol and hydrometeor scattering and absorption are computed with the Wiscombe (Wiscombe 1980)-Mie module.

HELEEOS provides coverage over the world's ocean regions based on the Surface Marine Gridded Climatology database, which is derived from the Comprehensive Ocean-Atmosphere Dataset (COADS). This database, which includes virtually all marine data from 1854 through 1997 summarized by $1^{\circ} \times 1^{\circ}$ latitude-longitude boxes by month, provides the mean, median, minimum, maximum, standard deviation, and mode for 14 selected elements from ship synoptic surface observations. HELEEOS currently extracts mean values for air temperature, sea temperature, pressure, relative humidity, and wind speed for over-ocean locations from those 14 available elements. The integration of the ANAM (Gathman et al. 1998) and the NSLOT (Frederickson et al. 2000) codes, both driven by parameters from the Surface Marine Gridded Climatology database, provides enhanced representation of atmospheric effects over all ocean regions on the $1^{\circ} \times$ $1^{\circ}$ grid.

A diverse array of aerosol vertical profiles is also available. There are 10 profiles defined using the Optical Properties of Aerosols and Clouds (OPAC; Hess et al. 1998) code, 3 Moderate-Resolution Atmospheric Transmission (MODTRAN) aerosol profiles (Shettle and Fenn 1979), and the wind speed-driven aerosol mixtures from ANAM. ANAM is the default aerosol profile for maritime sites with the OPAC and MODTRAN definitions available as options.

\section{b. High-energy laser performance comparisons in different environments}

In the current study, "peak irradiance" is the metric used to quantify system performance. Irradiance is the measurement of power per unit area $\left(\mathrm{W} \mathrm{m}^{-2}\right)$ delivered to a target surface. An increased irradiance value for a given ocean location indicates improved HEL performance. For this research, the actual value of the peak irradiance in a particular environment is not as important as how that value compares to the peak irradiance computed for the same engagement setup in a different environment. Typically, this comparison will be made through a ratio of the peak irradiance for a climatologically derived atmosphere to the peak irradiance for a standard set of atmospheric conditions.

In this paper, two types of atmospheric models are compared: a standard atmosphere profile that does not vary, and atmospheric profiles based on climatology that do vary temporally and spatially. The cornerstone of the 
first type of profile, the "standard profile," is the U.S. Standard Atmosphere, 1976. This widely used model cannot be used as the only atmospheric characterization for this study for several reasons. Foremost, it lacks a distinct atmospheric boundary layer. The atmosphere is assigned a logarithmic pressure profile, and the temperature is the same everywhere at the surface with specified lapse rates for the primary layers of the atmosphere. Moisture data are not representative at low altitudes for most of the earth's surface. Also, the standard atmosphere does not contain aerosol data or turbulence profiles. Thus, invoking the standard atmosphere to quantify HEL propagation effects can lead to physically unrealistic and nonspatially varying results. However, the standard atmosphere is well known, and it does allow for intuitive comparisons. For this study, standard profiles of parameters not included in the U.S. Standard Atmosphere, 1976, are combined with the standard atmosphere and are compared against the second type of profile - the climatological profile. This second profile uses meteorological, aerosol, and turbulence models that are temporally and spatially variable, allowing for more accurate modeling of the atmosphere. These improved modeling profiles lead to more realistic laser weapon system simulations.

\section{c. Establishment of global synoptic ocean temperature dataset}

To deduce SST departures from the long-term means established in the 143-yr COADS dataset, comparisons are made with a recently derived SST dataset that covers the period 1990-2008. This dataset was created at the Naval Postgraduate School (NPS) from the Global Temperature and Salinity Profile Program. Optimal spectral decomposition was required to process the raw dataset into a research-usable gridded format, as described below.

\section{1) Global Temperature And SAlinity PRofile Program}

The Global Temperature and Salinity Profile Program (GTSPP) is a joint effort of the International Oceanographic Data and Information Exchange committee (IODE) and the Joint Commission on Oceanography and Marine Meteorology (JCOMM) of the World Meteorological Organization (WMO) and the Intergovernmental Oceanographic Commission (IOC). GTSPP creates a timely global ocean temperature and salinity dataset of known quality in support of the World Climate Research Programme (WCRP). The development of GTSPP began in 1989, and the program went into operation in November 1990 (Sun et al. 2009).

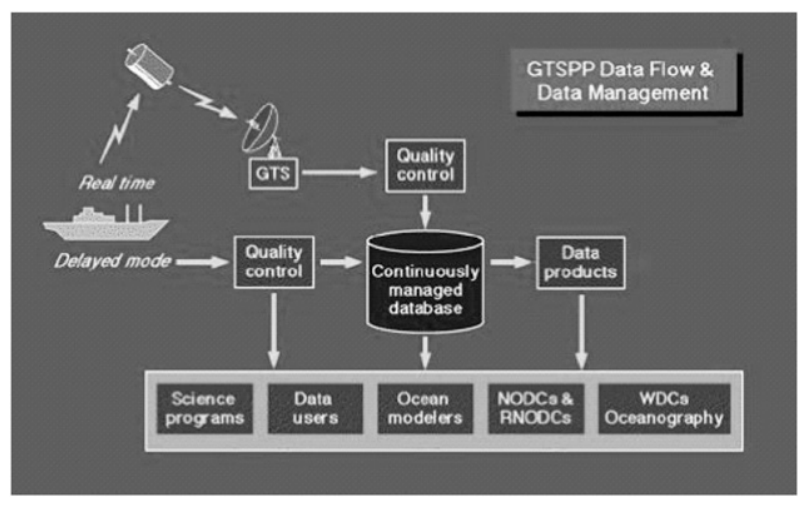

FIG. 1. A schematic diagram showing the data flow and management of the GTSPP (from Sun et al. 2009).

Currently, the GTSPP consists of three components: 1) the Global Telecommunication System (GTS) provided by the WMO for the transmission of oceanographic messages collected through various panels in the JCOMM program; the GTSPP uses this service to acquire real-time data, and real-time data processing services are provided by the Integrated Science Data Management (ISDM) service; 2) IODE Data Centers, where historical data are acquired either from other National Oceanographic Data Center (NODC) offices, or from cooperation with projects such as the Climate Variability and Predictability (CLIVAR) program, the World Ocean Database (WOD), and the Ship of Opportunity Programme (SOOP); and 3) the Continuously Managed Database (CMD). The NODC provides dataprocessing services for historical data and maintenance of the CMD (also known as the GTSPP archive). Historical data include both low-resolution data from the GTS and the full-resolution data from expendable bathythermographs (XBTs) or conductivity-temperaturedepth instruments (CTDs) from the ships that provided the real-time low-resolution data to the GTS, or fully processed and quality controlled data from other organizations (Fig. 1).

The GTSPP data contain upper-ocean temperature and salinity data and come mainly from profiling floats, XBTs, CTDs and bottles. A dramatic change occurred in mid-1999 with the initiation of the Argo Project; this marked the beginning of the use of temperature, salinity, and current reports (TESACs) to record profiles from robotic profiling floats. It is clear that the majority of the data were from XBTs (which primarily report profiles with temperature only) up until 1999, when the Argo Project began to report temperature and salinity profiles from profiling floats. During the period 2007-08, the number of bathythermographs (BATHYs) reported steadily increased from 24855 in 2007 to 27775 in 2008, 
while the number of TESACs increased markedly from 821321 to 1630360 between 2007 and 2008. A new dataset of 6869 CTD profiles (as of December 2008) derived from marine mammals was made available for the first time beginning in July 2008. These data are useful because they provide a high volume of data from areas between $60^{\circ}$ and $70^{\circ} \mathrm{S}$, a latitude band in which data from other sources are very sparse.

\section{2) OPTIMAL SPECTRAL DECOMPOSITION}

Sparse and noisy ocean GTSPP data need to be reanalyzed and mapped to the grid points before being assimilated into numerical models. Any variable including temperature can be decomposed into generalized Fourier series using the optimal spectral decomposition (OSD) method. The three-dimensional variable is then represented by linear combination of the products of the basis functions (or modes) and corresponding Fourier coefficients. If a rectangular closed ocean basin is considered, the basis functions are sinusoidal functions. If a realistic ocean basin is considered, the basis functions are the eigenfunctions of the three-dimensional Laplace operator with real topography. The Fourier coefficients are determined from observational data through solving a set of linear algebraic equations. The major benefit of using the OSD method is that the boundary conditions for the ocean variables (temperature, salinity, velocity) are always satisfied.

Let $(x, z)$ be horizontal and vertical coordinates and $t$ be time. A variable $c(\mathbf{x}, z, t)$ at depth $z_{k}$ is decomposed using the generalized Fourier series (Chu et al. 2003a,b, 2005a,b):

$c\left(\mathbf{x}, z_{k}, t\right)=A_{0}\left(z_{k}, t\right)+\sum_{m=1}^{M} A_{m}\left(z_{k}, t\right) \Psi_{m}\left(\mathbf{x}, z_{k}\right), \quad \mathbf{x} \in R\left(z_{k}\right)$,

where $M$ is the truncated mode number; $\Psi_{m}\left(\mathbf{x}, z_{k}\right)$ and $A_{m}\left(z_{k}, t\right)$ are the orthogonal basis functions (or called modes) and the spectral coefficients, respectively; and $R\left(z_{k}\right)$ is the area bounded by the lateral boundary $\Gamma\left(z_{k}\right)$ at $z_{k}$. The basis functions $\left\{\Psi_{m}\left(\mathbf{x}, z_{k}\right)\right\}$ are eigenfunctions of the horizontal Laplace operator with the basin geometry and certain physical boundary conditions. For temperature and salinity, the homogeneous Neumann boundary condition is taken at the solid boundary $\Gamma(z)$ (i.e., no heat and salt fluxes):

$$
\nabla_{h}^{2} \Psi_{m}=-\lambda_{m} \Psi_{m},\left.\quad \mathbf{n} \cdot \nabla_{h} \Psi_{m}\right|_{\Gamma}=0, \quad m=1,2, \ldots, M,
$$

where $\nabla_{h}^{2} \equiv \partial^{2} / \partial x^{2}+\partial^{2} / \partial y^{2}$ and $\mathbf{n}$ is the unit vector normal to $\Gamma(z)$. The basis functions $\left\{\Psi_{m}\right\}$ are independent of the data and therefore are available prior to the data analysis. The OSD method has two important procedures: optimal mode truncation and determination of spectral coefficients $\left\{A_{m}\right\}$. After the two procedures, the generalized Fourier spectrum (1) is used to provide data at regular grids in space and time.

The optimal mode truncation number $M_{\mathrm{opt}}$ is defined as the critical mode number with the set of spectral coefficients $\left\{A_{m}\right\}$ least sensitive to observational data sampling and noise. For a sample size of $P$ and a mode truncation of $M$, the spectral coefficients $\left\{A_{m}\right\}$ are estimated by the least squares difference between the observed and calculated values:

$$
\begin{aligned}
J_{\mathrm{emp}} & =J\left(\tilde{A}_{1}, \ldots, \tilde{A}_{M}, P, M\right) \\
& =\frac{1}{P} \sum_{j=1}^{P}\left[c^{(j)}-\sum_{m=1}^{M} \tilde{A}_{m}(z, t) \Psi_{m}^{(j)}(\mathbf{x}, z)\right]^{2} \rightarrow \min ,
\end{aligned}
$$

where the tilde represents the estimated value at $(\mathbf{x}, t)$. For homogeneously sampled data with low noise and without systematic error, the empirical cost function $J_{\text {emp }}$ should tend to 0 monotonically as $M$ increases to infinity. The set of the spectral coefficients $\left\{A_{m}\right\}$ depends on the mode truncation $M$. Optimal estimation of $\left\{A_{m}\right\}$ is equivalent to the determination of $M_{\mathrm{opt}}$ (Chu et al. 2003a,b). Generally, $M_{\text {opt }}$ is taken as $30-40$ for the basin-scale analysis (Chu et al. 2007).

Determination of the spectral coefficients is achieved by solving a set of linear algebraic equations of $\left\{\tilde{A}_{m}(z, t)\right\}$ after mode truncation:

$$
\mathbf{A} \hat{\mathbf{a}}=\mathbf{Q Y}
$$

where $\hat{\mathbf{a}}$ is the estimated state vector ( $L$ dimensional) for the exact state vector $\mathbf{a}, \mathbf{A}$ is a $P \times L$ coefficient matrix, $\mathbf{Q}$ is a $P \times P$ square matrix $(P>L)$, and $\mathbf{Y}$ is a $P$-dimensional observation vector, consisting of a signal $\overline{\mathbf{Y}}$ and a noise $\mathbf{Y}^{\prime}$,

$$
\mathbf{Y}=\overline{\mathbf{Y}}+\mathbf{Y}^{\prime}
$$

Because of the high level of noise contained in the observations, the set of algebraic equations is ill-posed and needs to be solved by a regularization method. Nonsingular orthogonal transformation is conducted through multiplication of (4) by a plane rotation matrix $\mathbf{S}$ from the left (Chu et al. 2004),

$$
\text { SAâ }=\mathbf{S Q Y},
$$

which changes the coefficient matrix and the source term from (A, QY) to (SA, SQY) and provides the 


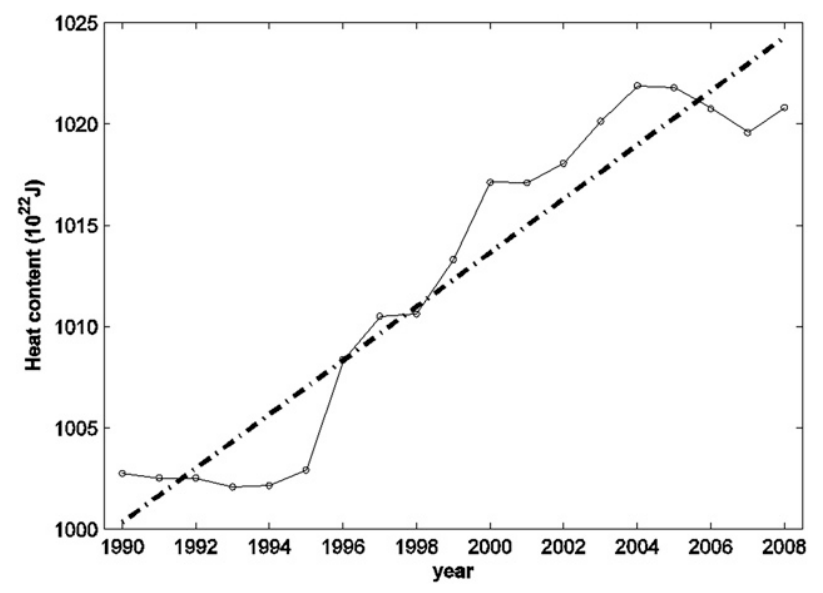

FIG. 2. Time series of yearly ocean $H_{C}\left(10^{22} \mathrm{~J}\right)$ for the upper layer $(0-700 \mathrm{~m})$.

opportunity to minimize the imperfection of the new system (6), which can be solved by the usual algebraic methods such as the Gauss method.

\section{3) Global gridded oceAn temperature DATASET}

More than 6 million temperature profiles (XBT, CTD, Argo, ...) from GTSPP were transferred from NOAA/ NODC to NPS. Each profile contains several hundred data points. For any given month the original data size is 822 MBytes. Our main objective is to provide the scientific and operational communities with three-dimensional ocean fields (such as temperature and salinity) that have sufficient resolutions in space $\left(1^{\circ} \times 1^{\circ}\right)$ and time $(1$ month $)$ that can effectively represent the rapid climate change. Such a three-dimensional global synoptic temperature and salinity dataset was established from January 1990 to December 2008. For example, yearly ocean heat content $H_{C}$ for the upper layer $(0-700 \mathrm{~m})$ was computed from the dataset by

$$
H_{C}(t)=\int_{-H}^{0} \int_{x} \int_{y} \rho c_{p} T(x, y, z, t) d x d y d z
$$

where $(x, y)$ and $z$ are horizontal and vertical coordinates, $H=700 \mathrm{~m}$, and $T$ is the yearly temperature. The horizontal integration is over the whole ocean domain. The upper-ocean $H_{C}$ has an evident upward trend with a rate nearly $1.34 \times 10^{22} \mathrm{~J} \mathrm{yr}^{-1}$ from 1990 to 2008 (Fig. 2).

\section{Methodology}

Using the HELEEOS model, low-altitude laser engagements are simulated to study atmospheric effects on the employment and operation of military weapon systems. The focus in the current study is on SSTs and marine optical turbulence effects.

HELEEOS currently relies on the Surface Marine Gridded Climatology database, which is derived from the 143-yr COADS dataset. This study compares the expected HEL performance based on these Surface Marine Gridded Climatology data and the new SST data obtained from the NPS. The new data are organized by latitude, longitude, month, and year and cover 19902008. Monthly averages are calculated over the $19 \mathrm{yr}$ to generate new SST means (Fig. 3). These means are then used by HELEEOS to model the expected maritime HEL performance.

Engagement and geometry parameters for the current study include the following:

- 20-m platform altitude, $10 \mathrm{~m} \mathrm{~s}^{-1}$ horizontal velocity, heading west $\left(270^{\circ}\right.$ true);

- $90^{\circ}$ relative azimuth to the target (laser pointing north);

- 5-m target altitude, $310 \mathrm{~m} \mathrm{~s}^{-1}$ horizontal velocity, heading south $\left(180^{\circ}\right.$ true);

- 5000-m slant range;

- 1.0642- $\mu$ m wavelength;

- summer and winter (characterized by January and July data);

- a Bufton wind profile, which assumes a $10 \mathrm{~m} \mathrm{~s}^{-1}$ ground speed from the west and an increase in speed slightly with altitude-this defines the "natural wind"; and

- clear conditions with no clouds or rain.

This particular low-altitude engagement scenario, with its combination of the natural crosswind and the motion of the platform both perpendicular to the laser line of sight and into the natural wind, minimizes thermal blooming — essentially "cooling" the laser beam-to highlight the impacts of marine optical turbulence. Thermal blooming occurs when energy at the wavelength the laser is operating at is absorbed by atmospheric constituents. This results in heating of the beam path and a corresponding reduction in the value of the refractive index of the air. This in turn results in a negative lens effect along the path and causes the beam to diverge. Thermal blooming is a nonlinear effect and can dramatically decrease the effectiveness of a laser weapon system. The climatological conditions are further defined by the NSLOT turbulence model, heavily driven by the air-sea temperature difference, and the ANAM model. For the most part, the datasets that are compared in this study are very similar except that the baseline SST means in the Surface Marine Gridded Climatology database ("old" data) are replaced with the new 19-yr SST means calculated from the NPS data ("new" data). All other conditions such as air temperature and relative humidity remained as 

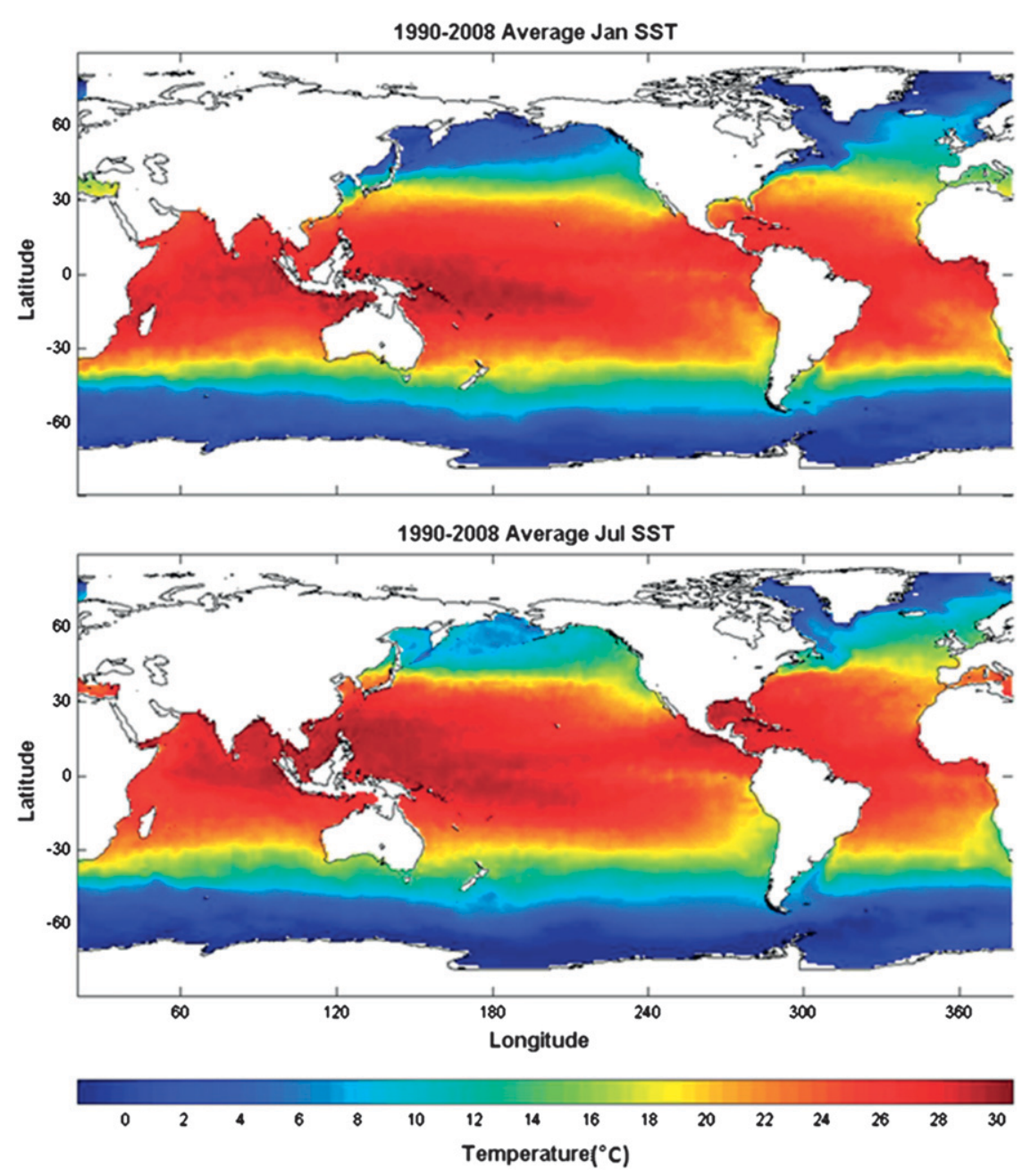

FIG. 3. Monthly mean SST calculated from the gridded GTSPP data from 1990 to 2008 during (top) January and (bottom) July.

defined in the Surface Marine Gridded Climatology database.

Turbulence at all scales creates temperature gradients that cause variations in the index of refraction. For smaller-scale turbulence $(<100-\mathrm{m}$ length scale, such as that created in the maritime surface layer by air-sea temperature differences and surface ocean waves), visible and infrared (IR) light will have different "optical distances" to travel over fairly short paths. This leads to changes in the phase of the light. Phase aberrations, when propagating through space, lead to changes in intensity of the light beam. Small-scale turbulence as it applies to visible and near-IR light or laser propagation is typically called optical turbulence (Perram et al. 2010). Optical turbulence is often quantified with the index of refraction structure constant $C_{n}^{2}$. This parameter has a fairly significant wavelength dependence (Fiorino et al. 2009); for example, a microwave radar at $\sim 10$-cm wavelength would measure a different $C_{n}^{2}$ value than a scintillometer operating at $\sim 1 \mu \mathrm{m}$ would measure for the same optical path. This is due to the microwave energy being sensitive to both temperature and humidity gradients, while the near-IR light is mainly only affected by temperature gradients. Additionally, focused, near-IR laser energy is most affected by optical turbulence on the same scale or smaller than the laser aperture diameter (typically less than $1 \mathrm{~m}$ ). Thus, turbulent disturbances larger than $1 \mathrm{~m}$, such as those produced by sea surface waves (Hristov 2008), are not a significant component of the $C_{n}^{2}$ parameter for the nearIR laser propagation modeled in this study.

Peak irradiance delivered to the target is calculated for the old and new climatological conditions as defined above. For the comparison ratios, a standard irradiance value is determined by HELEEOS by using the above engagement geometry and the following standard conditions: 

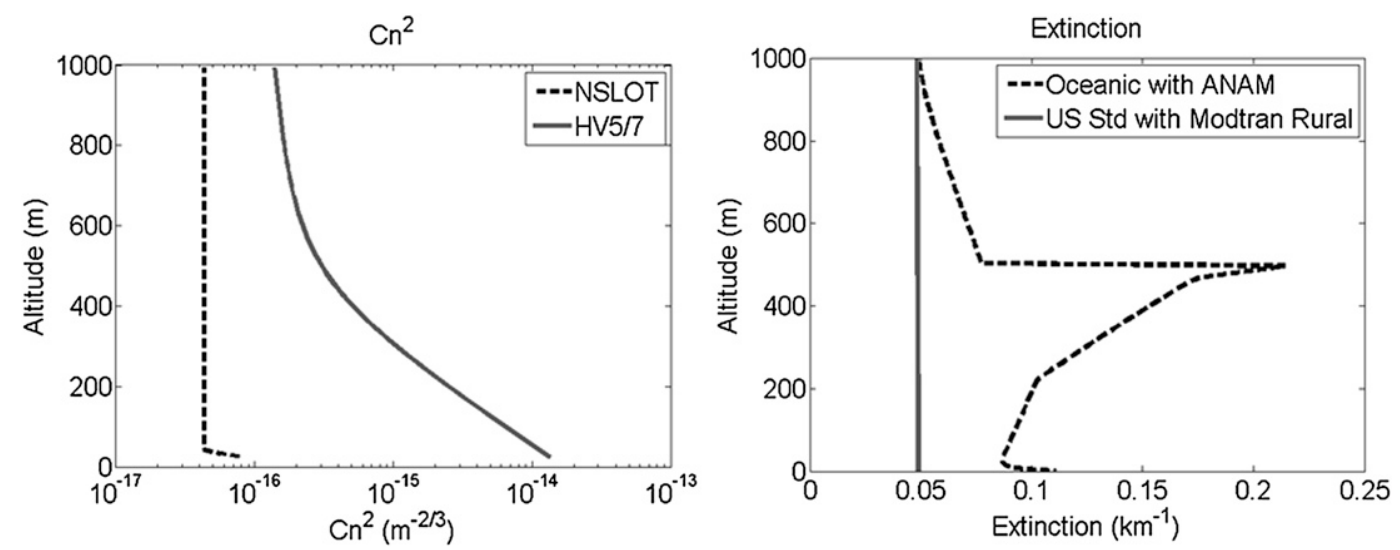

FIG. 4. Comparison of (left) optical turbulence strength and (right) atmospheric extinction for $1.0642 \mu \mathrm{m}$ for climatological (dashed) and standard conditions (solid), between the surface and 1000-m altitude for 50th percentile $\mathrm{RH}$, daily average, molecular, and aerosol effects only, for a maritime location off the eastern coast of Africa $\left(11.5^{\circ} \mathrm{N}\right.$, $58.5^{\circ} \mathrm{E}$ ). The climatological conditions are based on NSLOT for optical turbulence and COADS with ANAM aerosols for extinction. The standard conditions are based on Hufnagel-Valley $5 / 7$ for turbulence and U.S. Standard Atmosphere, 1976, with MODTRAN rural aerosols for extinction.

U.S. Standard Atmosphere, 1976, a rural MODTRAN aerosol model, and the Hufnagel-Valley $5 / 7$ optical turbulence model. Hufnagel-Valley $5 / 7$ is a long standing and widely used vertical profile model of optical turbulence, specifically at a wavelength of $0.55 \mu \mathrm{m}$, for which the Fried coherence length for a vertical path from the earth's surface to space is $5 \mathrm{~cm}$ and the isoplanatic angle is $7 \mu \mathrm{rad}$ (Hufnagel 1985). The Hufnagel-Valley $5 / 7$ profile is defined by the following equation, which is based on extensive overland thermosonde measurements:

$$
\begin{aligned}
C_{n}^{2}(h)= & 5.94 \times 10^{-53}\left(\frac{W}{27}\right)^{2} h^{10} e^{-h / 1000} \\
& +2.7 \times 10^{-16} e^{-h / 1500}+A e^{-h / 100},
\end{aligned}
$$

where in this case $h$ is the height (m), $W$ is the highaltitude wind speed, and $A$ is the assumed $C_{n}^{2}$ surface value. Typical values for $W$ and $A$, respectively, are $21 \mathrm{~m} \mathrm{~s}^{-1}$ and $1.7 \times 10^{-14} \mathrm{~m}^{-2 / 3}$.

The impacts of the atmospheric boundary layer effects on the results at $1.0642 \mu \mathrm{m}$ are significant. Figure 4 compares low-altitude turbulence strength and extinction at $1.0642 \mu \mathrm{m}$ for both the old climatological and standard atmosphere assumptions for a location off the eastern coast of Africa. These plots provide insight into why results at $1.0642 \mu \mathrm{m}$ are significantly better when climatological conditions (old and new) are assumed for a large number of maritime locations. Climatologically based turbulence (old and new), calculated using NSLOT and driven primarily by often near-zero air-sea temperature difference is two orders of magnitude less than the Hufnagel-Valley $5 / 7$ result at these low altitudes and dominates the results at the shorter laser wavelengths for these extremely low-altitude geometries. NSLOT is a well-verified model (Frederickson et al. 2000) and is used in the simulations to define the $C_{n}^{2}$ values within the first $50 \mathrm{~m}$ of altitude. Above $50 \mathrm{~m}$, the NSLOT $C_{n}^{2}$ value is held constant at the 50-m value until intercepting the Hufnagel-Valley curve. The Hufnagel-Valley $5 / 7$ profile was developed from overland optical turbulence measurements and includes a "spike" in near-surface $C_{n}^{2}$ values to presumably represent daytime solar heating effects (Hufnagel 1985). The comparisons to NSLOT indicate it is not representative of most near-surface maritime conditions; however, there is no accepted maritime standard optical turbulence model, so HufnagelValley $5 / 7$ is used despite the misfit.

Climatologically based extinction is generally greater than that for the standard case. At the shorter wavelengths (such as $1.0642 \mu \mathrm{m}$ ), this extinction is primarily due to aerosol scattering. The right panel of Fig. 4 demonstrates how extinction varies in the boundary layer in the climatological atmospheres for $1.0642 \mu \mathrm{m}$. Because maritime aerosols are salt based and hygroscopic, they increase in size and cause more scattering with height within the boundary layer due to increasing relative humidity with height (Fiorino et al. 2007). The decrease of extinction with height in the first $20 \mathrm{~m}$ above the surface seen in the right panel of Fig. 4 is due to wind speed effects on the multimodal aerosol distributions within ANAM.

HEL performance is evaluated via analysis of the peak irradiance calculated from the old and new climatological conditions to the standard irradiance value. There are about 35000 maritime data points used in this study. This number of data points is achieved because 

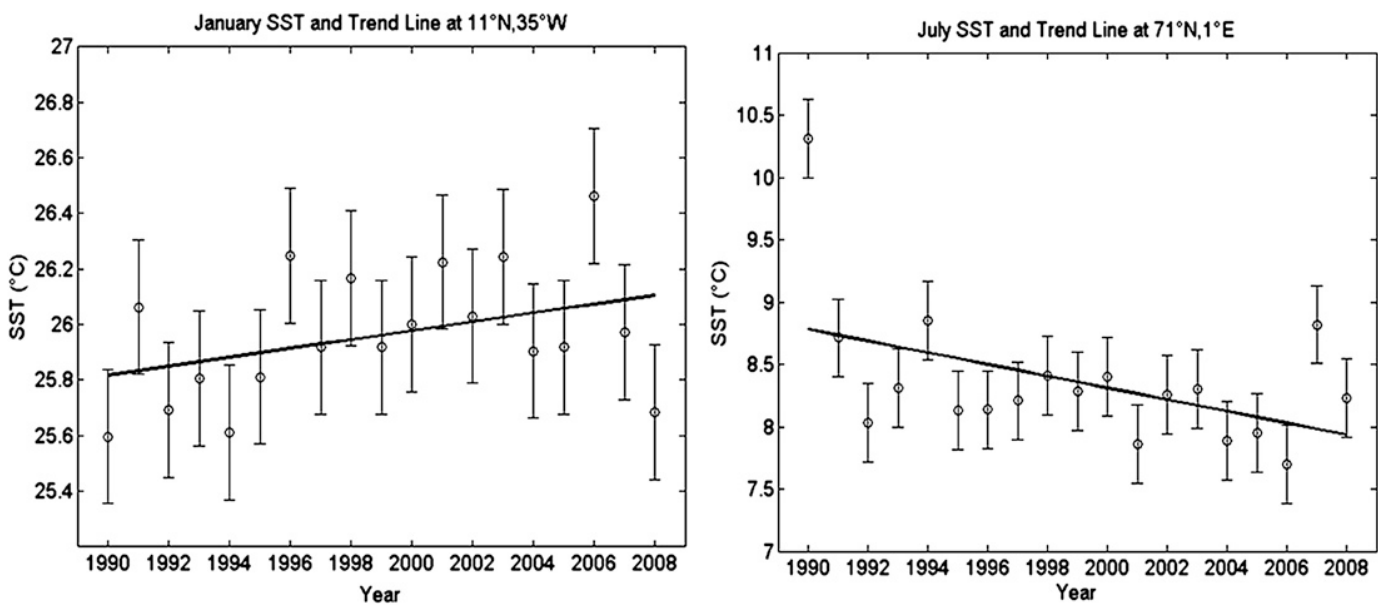

FIG. 5. Scatterplots of two locations showing new SST vs year with trend line. Trend lines at each grid point are used to project SSTs for years 2020 and 2050. Uncertainty bars are defined by the standard deviation about the 19-yr mean SST for that grid point.

both the Surface Marine Gridded Climatology database and the NPS data are on a $1^{\circ} \times 1^{\circ}$ latitude-longitude grid.

To enable a trend analysis and projection of potential SSTs, linear regressions are calculated using the new SST dataset. A simple regression equation in the form of $y=m x+b$ is used for each point of latitude and longitude. There are 19 data points that go into each calculation. For example, consider the data for the two grid points used in Fig. 5. For the month of January, there are 19 temperature measurements at each of these two points (1990-2008). Similarly, a separate regression is calculated for the data in July. Based on these regression equations, SST projections are made for 2020 and 2050.
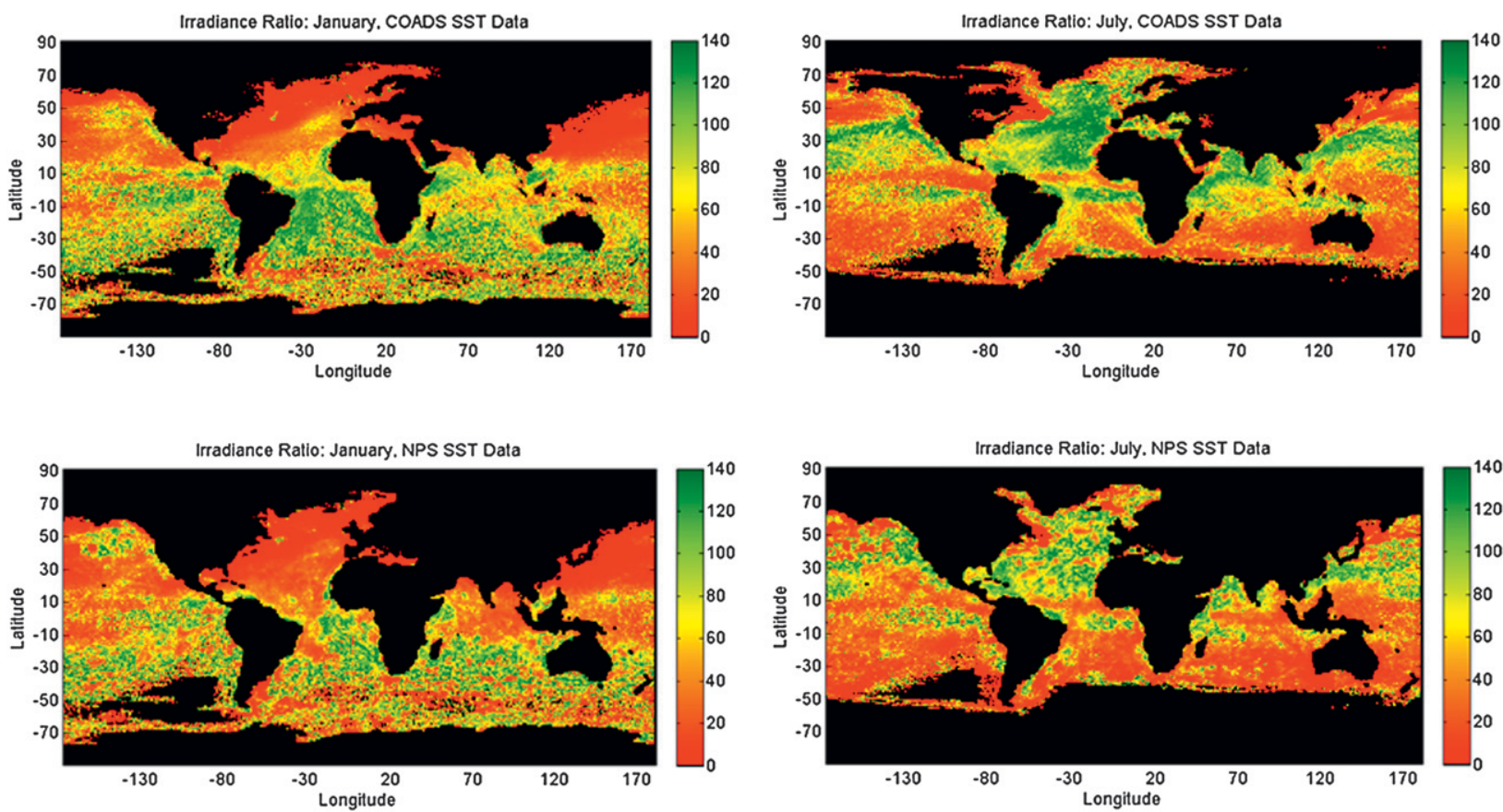

FIG. 6. Performance charts for January and July based on the old Surface Marine Gridded Climatology and the new SST data from NPS. Green indicates a high-valued ratio that implies much better performance in the climatological conditions than in the standard atmosphere conditions. Red indicates a lower-valued ratio that implies only slightly better performance in the climatological conditions than in the standard atmosphere conditions. These charts are based on the engagement geometry described above in section 2 . 

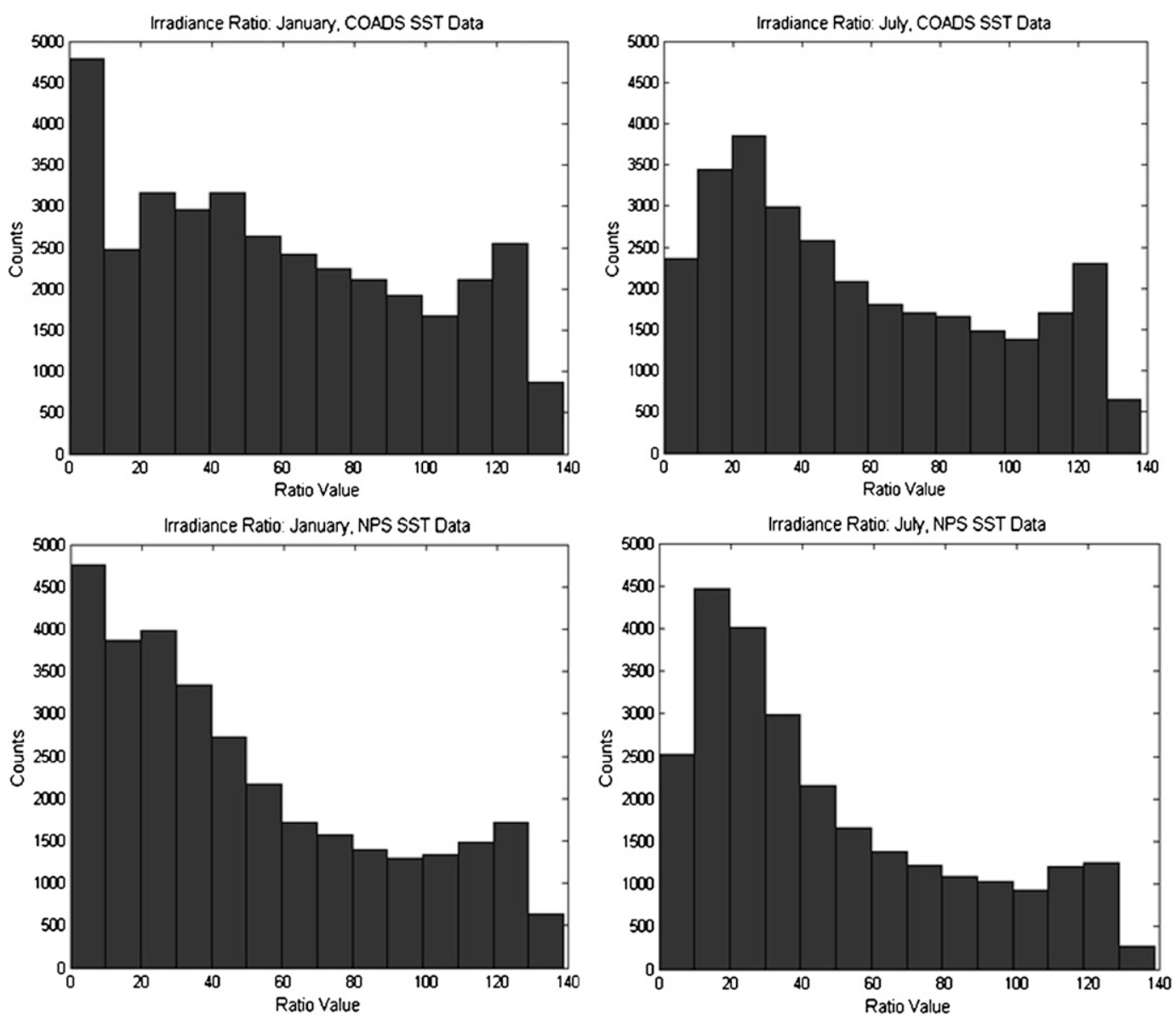

FIG. 7. Histograms of irradiance ratios for (left) January and (right) July of the (top) old Surface Marine Gridded Climatology calculations and (bottom) new SST data. Note the shift to lower-valued ratios in the histograms on the new dataset as compared with the old dataset.

The $R$-squared values of the linear regressions are low $(0.2-0.65)$, but a linear regression introduces the least amount of bias given the rather limited scatterplots of data. The example scatterplots and linear regression fits at two grid points in Fig. 5 are shown with uncertainty bars that are defined by the standard deviation about the 19-yr mean SST for that grid point. The projected data are used in HELEEOS with the same geometry/engagement parameters to model future (2020 and 2050) HEL performance.

\section{Results}

Comparisons are made between the irradiance ratios (climatological conditions/standard value) calculated from the old Surface Marine Gridded Climatology database and the new SST data from NPS. Over all maritime grid points, the differences are minor, but the number of lower-valued ratios is greater in the newer data from NPS as compared with the older Surface Marine Gridded Climatology data. This means the expected overall HEL performance does decrease slightly in the new SST scenario. Also, the irradiance calculated with a standard atmosphere is very conservative as compared to the irradiance calculated with a temporally and spatially varied climatological atmosphere (old or new). As a previous study has found (Fiorino et al. 2008), the assumption of standard atmosphere conditionsU.S. Standard Atmosphere, 1976, with $23-\mathrm{km}$ visibility MODTRAN rural aerosols and Hufnagel-Valley 5/7 optical turbulence-in modeling analyses can lead to significant, misleading deviations from conclusions derived using realistic (climatological) environmental conditions. The most notable "standard conditions" deviations from climatology in this study are the lack of boundary layer aerosol size and extinction variations and the inappropriateness of the Hufnagel-Valley $5 / 7 C_{n}^{2}$ profile in near-surface maritime environments. These deviations are apparent in our data; some irradiance ratios are higher than 100 in both the old and new datasets. This means that the irradiance obtained from 

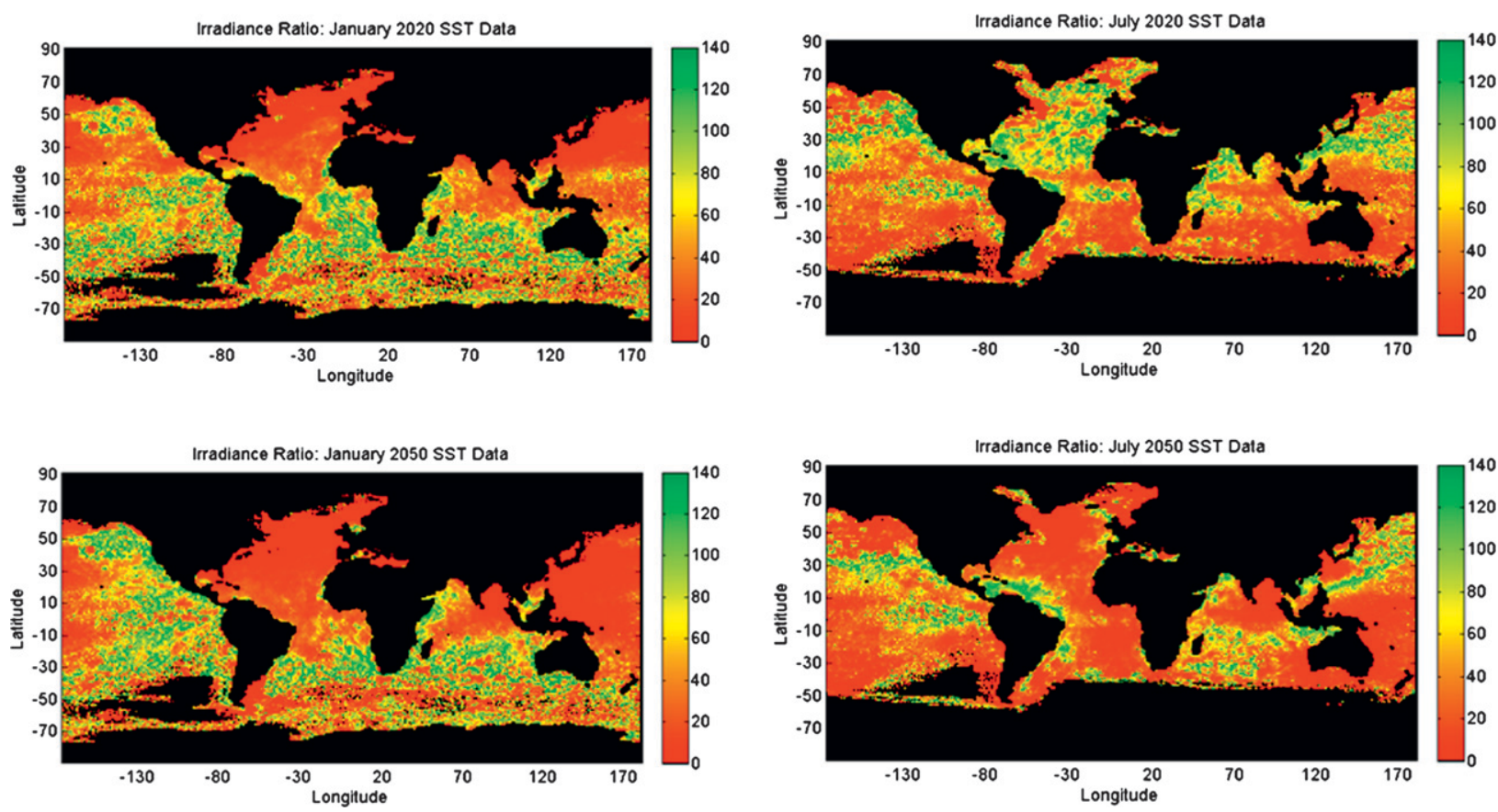

FIG. 8. Irradiance ratios for (left) January and (right) July (top) 2020 and (bottom) 2050. These irradiance ratios were calculated using the same geometry engagement as described for the old and new climatological plots. They rely on projected SST data from linear regressions like those shown in Fig. 5.

using a standard atmosphere can be as much as two orders of magnitude smaller than the climatologically based irradiance.

The global irradiance ratio charts are shown in Fig. 6 for January and July of the old Surface Marine Gridded Climatology and the new data from NPS. The top panels in Fig. 6 show the irradiance ratios for January and July based on data in the older Surface Marine Gridded Climatology database. The bottom panels in Fig. 6 show irradiance ratios for January and July calculated using the newer SST data from NPS. Generally, the results from the two databases follow the same patterns. Performance is better in the Southern Hemisphere in January as indicated by higher ratios, and in the Northern Hemisphere in July for both databases. This is primarily because air-sea temperature differences that cause more surface layer turbulence are generally greater in winter, and there is less aerosol scattering in summer due to lower relative humidity. The green areas (irradiance ratios $>\sim 90$ ) tend to be more broken up or not as "solid" in the charts from the newer dataset, but the overall change is very small. The overall mean irradiance ratio value calculated using the old Surface Marine Gridded Climatology is $\sim 56$, and the mean irradiance ratio value calculated using the new SST data from NPS is $\sim 47$ for a difference of $16 \%$. Modeled performance is better in the standard conditions rather than the climatological conditions (irradiance ratios $<1$ ) in only $\sim 2 \%$ of the January cases (both new and old), and $\sim 1 \%$ of the July cases (new and old). Histograms of the values plotted in Fig. 6 are shown in Fig. 7.

By using the projected SST data, future HEL performance is modeled using HELEEOS. The irradiance ratio charts for 2020 and 2050 are shown in Fig. 8, and the difference between the 2020 and 2050 levels of performance and the current level of performance is shown in Fig. 9. The 2020 and 2050 performance comparisons to current conditions are made by subtracting the old climatological irradiance ratios (top plots in Fig. 6) from the 2020 or 2050 irradiance ratios. Figure 10 shows histograms of the 2020 irradiance ratio-old data irradiance ratio differences for January and July. Only slight differences are discernible in the performance for 2020 as compared to the current performance, as is evidenced by the large amount yellow, near-zero difference areas depicted in the top panels of Fig. 9, and the near-zero peaks in the histograms of Fig. 10. However, when the gridpoint trends are projected another $30 \mathrm{yr}$ to 2050, the comparison to current conditions shows the expected performance degradation over large areas of the North Atlantic and Bay of Bengal in July, and the South Atlantic in January. This is evident in the red-colored regions seen in the bottom panels of Fig. 9. It is important to note that these performance changes are based on the assumption 

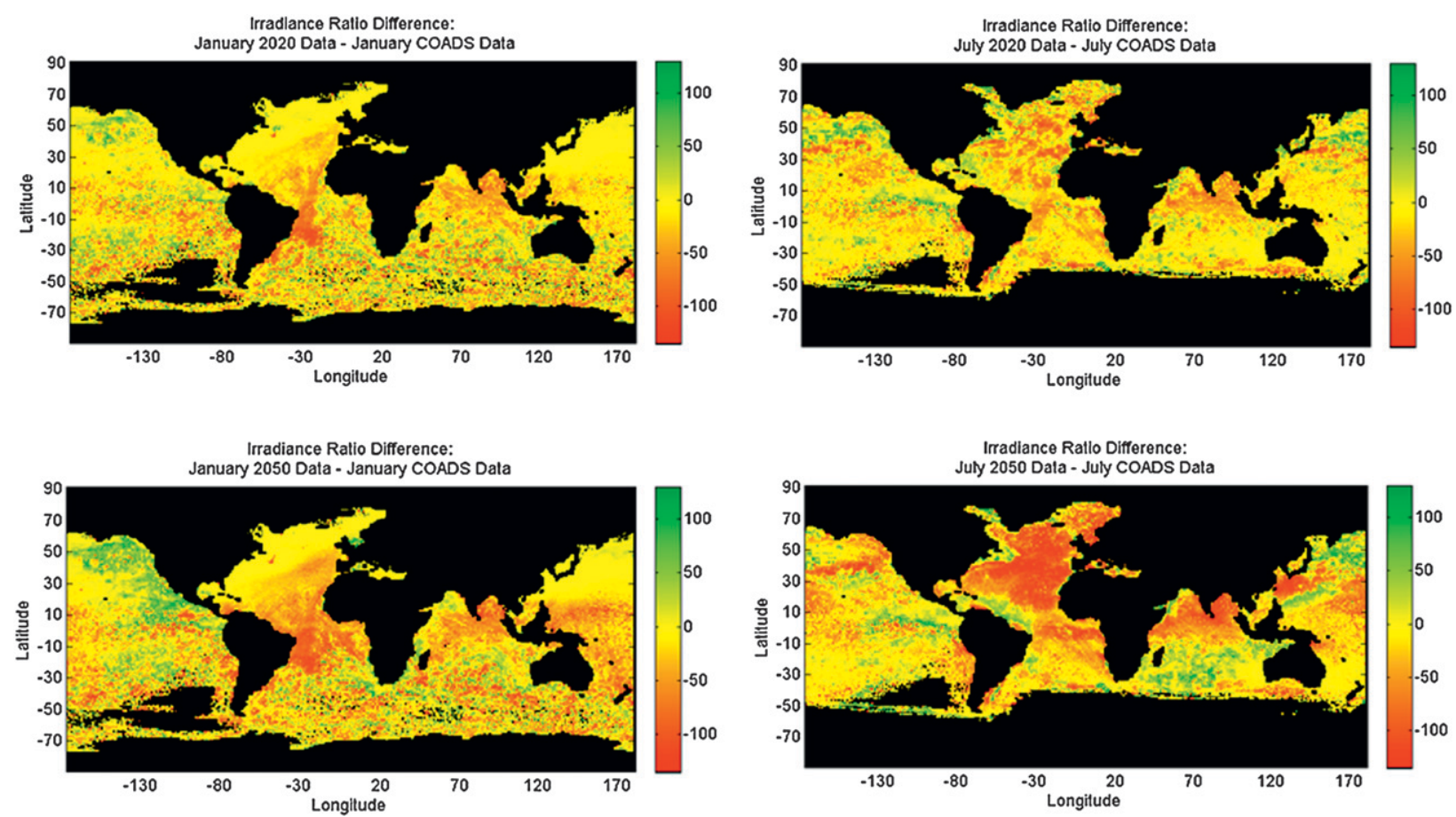

FIG. 9. Irradiance ratios differences for (left) January and (right) July. These irradiance ratio differences were calculated by subtracting the irradiance ratio value from the old climatological database from the (top) 2020 or (bottom) 2050 irradiance ratio. In general, a yellow color indicates little change in expected performance from current conditions to the projected year, green indicates improved expected performance, and red indicates degraded expected performance.

that the ocean temperatures will continue to follow the same trends at each point for the next 10-40 yr.

Roughly $60 \%$ of the irradiance ratios appear as yellow in the top (2020) panels of Fig. 9, a ratio difference of 25 or less. Most areas show little discernable change. Some areas, such as the North Pacific in January, the Korean Peninsula in July (Fig. 11, right), and some coastal regions around Asia and Africa in July do show a slight
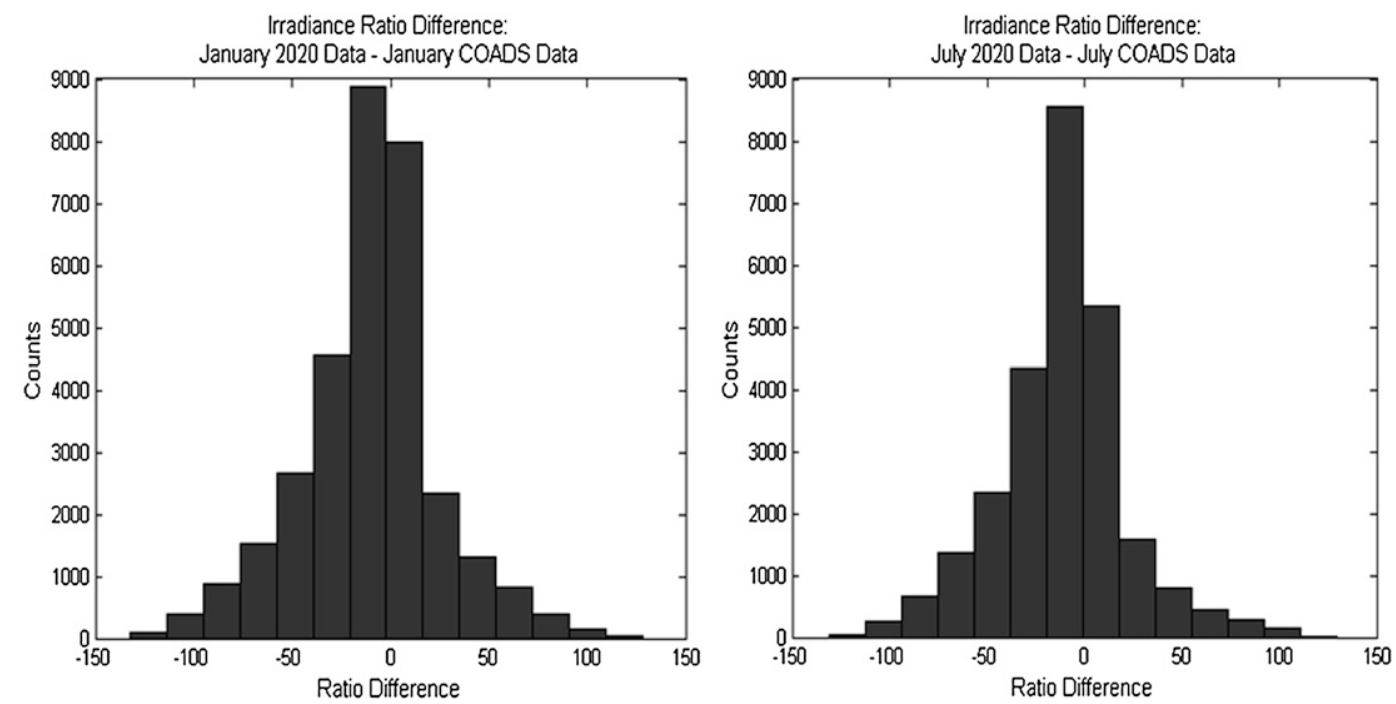

FIG. 10. Irradiance ratio differences: 2020 performance - current (old data) performance with histograms. The difference was found by subtracting the performance calculated using the Surface Marine Gridded Climatology database from the predicted performance in 2020 based off the projected SST data from NPS. The histograms of the irradiance difference show over 7000 data points are centered around zero. 

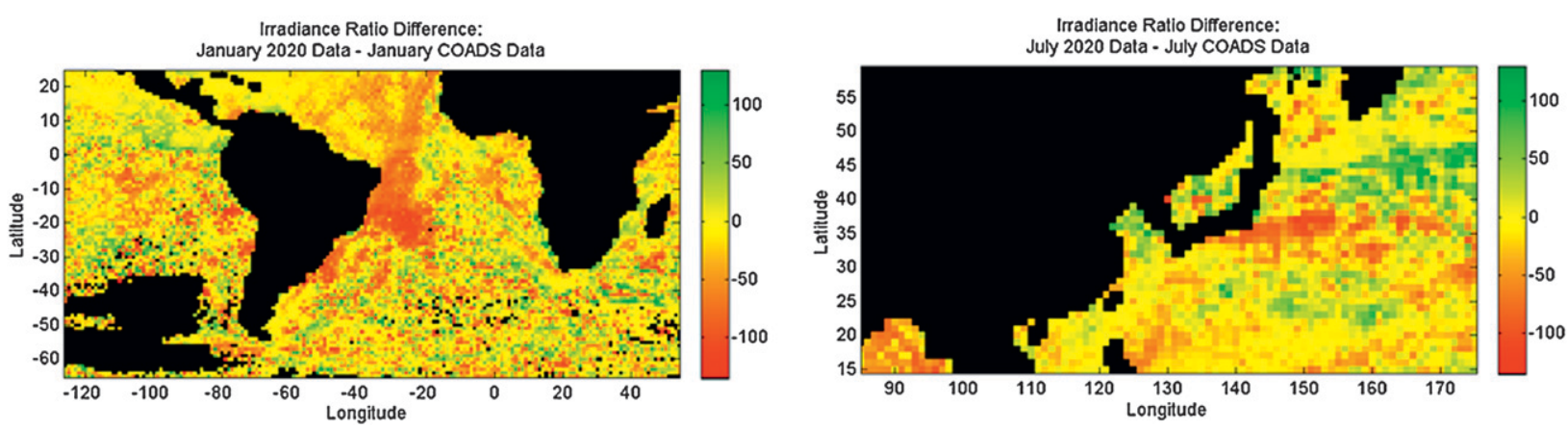

FIG. 11. Zoomed-in views of (left) South America in January and (right) the Korean Peninsula in July showing the difference between the irradiance ratio for the year 2020 and now. Note the poorer than expected performance off the coast of South America but the better than expected performance around the Korean Peninsula. These plots are zoomed-in views of the top panels in Fig. 9.

improvement; however, many areas show little change. The eastern coast of South America in January (see Fig. 11, left) and the Atlantic and Indian Oceans in July show the largest decreases in performance. The irradiance ratio differences in these regions constitute most of the nonzero values shown in Fig. 10.

The main driving force behind the changes in HEL performance is the air-sea temperature difference. Airsea temperature difference is the primary variable that determines the surface layer turbulence that can dramatically affect laser propagation in the lower atmosphere near the sea surface. Air temperature data are available in the Surface Marine Gridded Climatology database and are used by HELEEOS in calculations. However, air temperature is not varied in this study. Therefore, any change in SST directly results in a change in the air-sea temperature difference. These changes in air-sea temperature difference either increase or decrease deleterious atmospheric effects on expected HEL performance. This identifies the main reasoning for not allowing the air temperature to vary in response to the ocean surface temperature changes-it maximizes the differences to simulate the worst-case scenario. Figure 12 shows the expected air-sea temperature differences for January and July 2020 with this assumption applied.

Average SST was calculated for the Surface Marine Gridded Climatology database and for the new data from NPS. The global SST average for the Surface Marine Gridded Climatology database was found to be $17.3^{\circ} \mathrm{C}$. The global SST average for the new data from NPS was found to be $16.1^{\circ} \mathrm{C}$. It should be noted, however, that the COADS-derived Surface Marine Gridded Climatology database spans a much longer time period (143 yr) and includes more observations than the new dataset from NPS, which spans only $19 \mathrm{yr}$. To find these averages, only January and July of each dataset were used. In the new dataset, there are a few points that are significantly cooler than the COADS data for the same points, thereby lowering the global mean, but most points are slightly warmer and exhibit trend lines with small positive slopes, as shown on the left in Fig. 5.

\section{Conclusions}

The overall conclusion of this study-where anticipated SST change is used to drive marine surface layer optical turbulence change that could impact maritime high-energy-laser engagement scenarios-is that the effects of the anticipated SST changes would in general be minor or negligible in most oceanic locations. The analysis of the COADS dataset versus the 19-yr NPS dataset did indicate a very slight decline in expected HEL performance in the scenario studied at a number of locations, but most areas showed little discernible change.

Analysis of the COADS data versus the NPS SSTs does show that the air-sea temperature difference has become slightly more negative on a world-wide scale. At most points, this means SSTs are slightly warmer in the 19 -yr database than in the 143-yr database. Notably, the change in SSTs reduces the air-sea temperature difference in some areas (e.g., around the Korean Peninsula in July), thus leading to a slight improvement in anticipated HEL weapon system performance based on the NPS data over the COADS dataset.

For the portion of this study where the differences between the COADS and NPS datasets are analyzed for trends and projected to 2020 and 2050 scenarios, the vast majority of the oceanic locations show the changes in expected HEL performance to be insignificant. Specifically, the projection analysis indicates areas in the South Atlantic to have the largest decrease in expected performance, while the North Pacific shows the greatest increase in expected performance in 2020 and 2050. The histograms show that the frequency of lower-valued irradiance ratios increases for 2020, suggesting more 

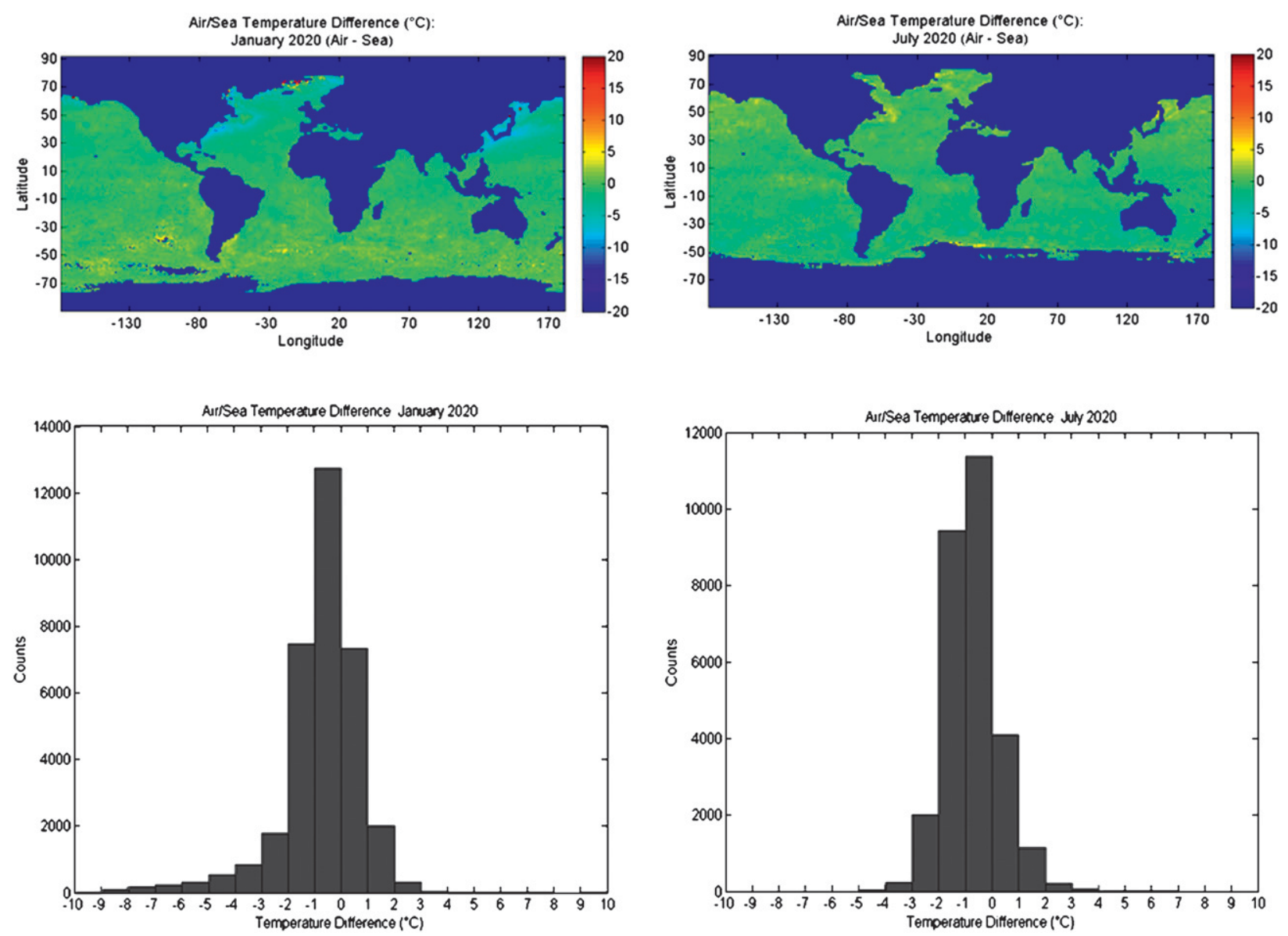

FIG. 12. Air-sea temperature difference for (left) January and (right) July 2020. (top) The air-sea temperature difference plots show that the majority of points exhibit little change (green shades). (bottom) The histograms show that most points indicate a slightly more negative air-sea temperature difference, meaning that ocean temperature increased slightly at most grid points.

locations experience a slight decrease in anticipated HEL system performance than an increase. On a global scale, the air-sea temperature difference continues to become more negative, meaning sea temperatures increase slightly. However, air temperature was not varied in this worst-case sensitivity study, so any change in SST will directly result in a change in the air-sea temperature difference.

Acknowledgments. The authors gratefully acknowledge the support of the High Energy Laser Joint Technology Office in development of the underlying models. They also thank Professor Daniel A. Nussbaum in the Operations Research Department at NPS and the USAF QDR Office for funding support. Additionally, the authors are indebted to the insightful comments and suggested improvements offered by two unnamed reviewers. The views expressed in this paper are those of the authors and do not necessarily reflect the official policy or position of the U.S. Air Force, U.S. Navy, the Department of Defense, or the U.S. government.

\section{REFERENCES}

Bartell, R. J., and Coauthors, 2005: Methodology for comparing worldwide performance of diverse weight-constrained high energy laser systems. Laser Source and System Technology for Defense and Security, G. L. Wood, Ed., International Society for Optical Engineering (SPIE Proceedings, Vol. 5792), 76-87.

Bussey, A. J., J. R. Roadcap, R. R. Beland, and G. Y. Jumper, 2000: Master data base for optical turbulence research in support of airborne laser. AFRL-VS-TR-2000-1545, Air Force Research Laboratory, 33 pp.

Chu, P. C., L. M. Ivanov, T. M. Margolina, T. P. Korzhova, and O. V. Melnichenko, 2003a: Analysis of sparse and noisy ocean current data using flow decomposition. Part I: Theory. J. Atmos. Oceanic Technol., 20, 478-491.

,,,---- , and,$- 2003 \mathrm{~b}$ : Analysis of sparse and noisy ocean current data using flow decomposition. Part II: Applications to Eulerian and Lagrangian data. J. Atmos. Oceanic Technol., 20, 492-512. 
,-- , and -2004 : Rotation method for reconstructing process and fields from imperfect data. Int. J. Bifurcat. Chaos, 14, 2991-2997.

, — - and - 2005a: Seasonal variability of the Black Sea chlorophyll-a. J. Mar. Syst., 56, 243-261.

,- -, and O. V. Melnichenko, 2005b: Fall-winter current reversals on the Texas-Louisiana continental shelf. J. Phys. Oceanogr., 35, 902-910.

,,--- , and N. C. Wells, 2007: On long baroclinic Rossby waves in the tropical North Atlantic observed from profiling floats. J. Geophys. Res., 112, C05032, doi:10.1029/ 2006JC003698.

Fiorino, S. T., R. J. Bartell, G. P. Perram, D. W. Bunch, L. E. Gravley, C. A. Rice, Z. P. Manning, and M. J. Krizo, 2006: The HELEEOS atmospheric effects package: A probabilistic method for evaluating uncertainty in low-altitude high energy laser effectiveness. J. Dir. Energy, 1, 347-360.

,-- M. J. Krizo, and S. J. Cusumano, 2007: Expected worldwide, low-altitude laser performance in the presence of common atmospheric obscurants. J. Dir. Energy, 2, 363-375.

,,--- , and,- 2008 : Propagation variability assessments of ship defense HEL and HPM performance in worldwide maritime boundary layer environments at wavelengths of $1.0642 \mu \mathrm{m}, 2.141 \mu \mathrm{m}, 3.16 \mathrm{~mm}$, and $12.2 \mathrm{~cm}$. Atmospheric Propagation V, G. C. Gilbreath and L. M. Wasiczko, Eds., International Society for Optical Engineering (SPIE Proceedings, Vol. 6951), 69510G, doi:10.1117/12.777568.

,,--- , B. McClung, J. J. Cohen, R. M. Randall, and S. J. Cusumano, 2009: Broad spectrum optical turbulence assessments from climatological temperature, pressure, humidity, and wind. J. Dir. Energy, 3, 223-238.

Frederickson, P. A., K. L. Davidson, C. R. Zeisse, and C. S. Bendall, 2000: Estimates of the refractive index structure parameter (Cn2) over the ocean using bulk methods. J. Appl. Meteor., 39, 1770-1783.

Gathman, S. G., A. M. J. van Eijk, and L. H. Cohen, 1998: Characterizing large aerosols in the lowest levels of the marine atmosphere. Propagation and Imaging through the Atmosphere II,
L. R. Bissonnette, Ed., International Society for Optical Engineering (SPIE Proceedings, Vol. 3433), 41-52.

Hess, M., P. Koepke, and I. Schult, 1998: Optical properties of aerosols and clouds: The software package OPAC. Bull. Amer. Meteor. Soc., 79, 831-844.

Hristov, T., 2008: Statistical signature of the surface waves in the wind over the ocean. Preprints, 18th Symp. on Boundary Layers and Turbulence, Stockholm, Sweden, Amer. Meteor. Soc., 17A.3. [Available online at http://ams.confex.com/ams/ pdfpapers/139865.pdf.]

Hufnagel, R. E., 1985: Propagation through atmospheric turbulence. The Infrared Handbook, W. L. Wolfe and G. J. Zissis, Eds., Infrared Information Analysis Center, 6-1-6-56.

Koepke, P., M. Hess, I. Schult, and E. P. Shettle, 1997: Global aerosol data set. MPI Meteorologie Hamburg Rep. 243, $44 \mathrm{pp}$.

Perram, G. P., S. J. Cusumano, R. L. Hengehold, and S. T. Fiorino, 2010: An Introduction to Laser Weapon Systems. Directed Energy Professional Society, 473 pp.

Rothman, L. S., and Coauthors, 2005: The HITRAN 2004 modecular spectroscopic database. J. Quant. Spectrosc. Radiat. Transfer, 96, 139-204.

Shettle, E. P., and R. W. Fenn, 1979: Models for the aerosols of the lower atmosphere and the effects of humidity variations on their optical properties. AFGL-TR-79-0214, Air Force Geophysics Laboratory, 94 pp.

Squires, M. F., B. A. Bietler, S. T. Fiorino, D. L. Parks, F. W. Youkhana, and H. D. Smith, 1995: A method for creating regional and worldwide datasets of extreme and average values. Proc. 41st Annual Technical Meeting, Anaheim, CA, Institute of Environmental Sciences, 6-11.

Sun, C., and Coauthors, 2009: The data management system for the Global Temperature and Salinity Profile Programme. Proc. OceanObs'09, Venice, Italy, WMO, 8 pp. [Available online at http://www.nodc.noaa.gov/GTSPP/document/publications/ cwp4c14.pdf.]

Wiscombe, W. J., 1980: Improved Mie scattering algorithms. Appl. Opt., 19, 1505-1510. 